\title{
Maternal and perinatal outcome in pregnancy complicated by obstetric cholestasis: study from a tertiary care centre in North India
}

\author{
Deepika Jamwal*, Gurpreet Kour, Anil Mehta
}

Department of Obstetrics and Gynaecology, GMC Kathua, Jammu and Kashmir, India

Received: 12 May 2021

Accepted: 04 June 2021

\author{
*Correspondence: \\ Dr. Deepika Jamwal, \\ E-mail: deepikajamwal215@gmail.com
}

Copyright: (C) the author(s), publisher and licensee Medip Academy. This is an open-access article distributed under the terms of the Creative Commons Attribution Non-Commercial License, which permits unrestricted non-commercial use, distribution, and reproduction in any medium, provided the original work is properly cited.

\begin{abstract}
Background: Intrahepatic cholestasis of pregnancy (IHCP) is the most common pregnancy related liver disorder. It typically presents with troublesome itching and can lead to complications for both mother and foetus. Present study was carried out to study the incidence of Obstetric Cholestasis and its fetomaternal outcome in a tertiary care hospital.

Methods: It was a prospective epidemiological study during a period of one year (May 2020 to April 2021) over 120 pregnant ladies suffering from pruritus and detected as having Obstetric Cholestasis. They were followed up and maternal as well as perinatal outcome recorded. Appropriate statistical analysis done as applicable.

Results: The incidence of Obstetric Cholestasis in our hospital was 9.3\%. Majority of cases delivered at term (78.3\%). $41.6 \%$ patients delivered vaginally, $43.3 \%$ had emergency caesarean section, and $2.5 \%$ patients had instrumental delivery. Maternal morbidities are due to sleep disturbance (60\%), coagulation abnormality (13.3\%), increase chance of operative delivery $(55.8 \%)$ and postpartum haemorrhage $(12.5 \%)$. Neonatal complications include meconium aspiration (46.6\%), NICU admission (36.6\%), prematurity (5\%) and perinatal mortality (3.3\%).

Conclusions: Cholestasis of pregnancy causes maternal pruritus with impaired liver function tests. Maternal morbidity is increased in terms of increased caesarean section rates and discomfort due to pruritus. A timely intervention at 37-38 weeks will reduce the adverse perinatal outcome.
\end{abstract}

Keywords: Intrahepatic cholestasis of pregnancy, Pruritus

\section{INTRODUCTION}

Obstetric cholestasis (OC) or Intrahepatic cholestasis of pregnancy (ICP) is a cholestatic disorder characterized by unexplained pruritus during pregnancy with elevated serum bile acids $(>10 \mu \mathrm{mol} / \mathrm{L})$ and/or transaminases in late second and third trimester of pregnancy, in absence of other liver disease, and spontaneous resolution of signs and symptoms within two to three weeks after delivery. ${ }^{1,2}$

Obstetric cholestasis has been observed in almost all ethnic groups, but there is relevant geographical variation in its incidence. The incidence of OC among Indian women has been reported to be about $1 \% .^{3,4}$ Cholestasis of pregnancy is caused by an impairment of bile secretion in the liver.
As the bile backs up in the liver, the level of bile acids increases in the bloodstream. The bile acids are deposited in the maternal tissues like skin, causing intense pruritus (Heather Brannon, 2004). Obstetric cholestasis clinically manifests in the 2nd or 3rd trimester of pregnancy with generalized pruritus but without any skin rash (McDonald, 2002).

Pruritus begins in the palms and soles, with progression to the arms and legs, eventually, reaching the trunk and face. Pruritus is most severe at night. Jaundice is relatively uncommon, complicating only the most severe and prolonged cases (Milkiewiez et al., 2002). The condition worsens as pregnancy proceeds and there is spontaneous relief of symptoms and signs within two to three weeks 
after delivery. Intrahepatic cholestasis of pregnancy can have devastating consequences for the foetus with perinatal mortality reaching up to $11 \%$ to $20 \%$ in untreated cases. ${ }^{5}$ Adverse foetal outcomes associated with the conditions include preterm labour, preterm pre labour rupture of membranes, foetal distress, abnormal CTG, meconium staining, spontaneous intrauterine death. ${ }^{6,7}$

Ursodeoxycholic acid (UDCA) is considered to be a safe treatment option in the later part of pregnancy., ${ }^{8,9}$ The condition typically resolves within 48 hours of women giving birth with biochemical markers predominantly becoming normal within 2-4 weeks postnatally. ${ }^{10}$

Present study is aimed to detect the incidence of OC in our hospital and follow up those pregnancies to evaluate maternal and perinatal outcome.

\section{METHODS}

Our prospective epidemiological study was performed in the department of obstetrics and gynaecology in Government medical college Kathua in the union territory of Jammu and Kashmir over one year (May 2020 to April 2021). The study group included 120 patients which were enrolled from the outpatient department as well as from the patients admitted in the labour room with the history of intra hepatic cholestasis.

The diagnosis of obstetric cholestasis was based upon the clinical symptom of persistent pruritus without a skin rash associated with the biochemical evidence of mild to moderate cholestasis in the absence of any other liver disease, which resolved postnatally. Abnormal liver function was defined as at least 2-4 fold increase in transaminases not exceeding $250 \mathrm{IU} / \mathrm{ml}$ with or without mild increase in serum bilirubin not exceeding $5 \mathrm{mg} / \mathrm{dl}$.

Women with positive serology for Hepatitis A, B or C, previous history or sonographic evidence of gall bladder disease, PIH and those in whom liver function did not normalize within two weeks of delivery were excluded from this study. History taking, clinical examination and laboratory investigations were carried out to diagnose obstetric cholestasis. LFT was repeated every 2-4 weeks interval as required. All patients included in the study were given ursodeoxycholic acid 300-1200 mg/day in divided doses for the rest of the antenatal period. The data were collected in preformed proforma and written and informed consent was taken.

Maternal outcome was noted in terms of gestational age at delivery, mode of delivery and complications which includes sleep disturbance due to severe pruritus, deranged coagulation profile (increase PT), preterm labour, operative delivery and postpartum haemorrhage. Foetal outcome was studied in reference to prematurity, abnormal CTG, meconium stained liquor, NICU admission rate and perinatal death. Symptomatic relief of pruritus and liver function test was determined in all women two weeks after the delivery. The data obtained was entered in MS excel sheet and tabulated.

\section{RESULTS}

During the study period out of the 1280 antenatal registered women, 120 were diagnosed as having cholestasis of pregnancy giving an incidence of 9.3\%. 76 $(63.3 \%)$ were primigravida and 44 (36.6\%) were multigravidas. Majority of the patients were in the age group of 26-30 years (48.3\%) (Table 1). $78.6 \%$ patients delivered at term. $5 \%$ had preterm delivery (Table 2 ).

Table 1: Demographic analysis.

\begin{tabular}{|lll|}
\hline Variables & No. of cases & $\%$ \\
\hline Age & & \\
\hline$<20$ years & 8 & 6.6 \\
\hline 21-25 years & 38 & 31.6 \\
\hline 26-30 years & 58 & 48.3 \\
\hline$>30$ years & 16 & 13.3 \\
\hline Parity & & \\
\hline Primipara & 76 & 63.3 \\
\hline Multipara & 44 & 36.6 \\
\hline
\end{tabular}

Table 2: Distribution of patients according to gestational age at delivery.

\begin{tabular}{|lll|}
\hline Gestational age & No. of cases & $\%$ \\
\hline$<37$ weeks & 6 & 5 \\
\hline 37-40 weeks & 94 & 78.6 \\
\hline$>$ 40 weeks & 20 & 16.6 \\
\hline
\end{tabular}

Table 3: Distribution of patients according to liver function tests.

\begin{tabular}{|llll|}
\hline Variables & & No. of cases & $\%$ \\
Serum & $0.2-0.6$ & 58 & 48.3 \\
bilirubin & $0.6-1.0$ & 40 & 33.3 \\
(mg/dl) & $1.0-1.4$ & 16 & 13.3 \\
& $>1.4$ & 6 & 5 \\
\hline & $0-100$ & 46 & 38.3 \\
SGOT & $100-200$ & 52 & 43.3 \\
(IU/L) & $200-300$ & 15 & 12.5 \\
& $>300$ & 7 & 5.8 \\
\hline & $0-100$ & 54 & 45 \\
SGPT & $100-200$ & 44 & 36.6 \\
(IU/L) & $200-300$ & 17 & 14.16 \\
& $>300$ & 5 & 4.16 \\
\hline \multirow{2}{*}{ Serum } & $0-200$ & 18 & 15 \\
alkaline & $200-400$ & 48 & 40 \\
phosphatase & $400-600$ & 42 & 35 \\
& $>600$ & 12 & 10 \\
\hline
\end{tabular}

Jaundice was noticed in $18.3 \%$. Maximum $43.3 \%$ patients had SGOT in the range of 100-200. Maximum $45 \%$ patients had SGPT in the range of 0-100 (Table 3). 
Table 4: Distribution of patients according to mode of delivery.

\begin{tabular}{|lll|}
\hline Mode of delivery & No. of cases & $\%$ \\
\hline Elective CS & 15 & 12.5 \\
\hline Emergency CS & 52 & 43.3 \\
\hline Forceps & 3 & 2.5 \\
\hline Vaginal delivery & 50 & 41.6 \\
\hline
\end{tabular}

In the study group, $87.5 \%$ (105/120) women went into labour either spontaneously or after induction. $43.3 \%$ had emergency caesarean section and $41.6 \%$ patients delivered vaginally. $2.5 \%$ of the patients had instrumental delivery. Elective caesarean section rate was $12.5 \%$ (Table 4).

Table 5: Distribution of maternal outcome.

\begin{tabular}{|lll|}
\hline Variables & No. of cases & $\%$ \\
\hline Sleep disturbance & 72 & 60 \\
\hline $\begin{array}{l}\text { Deranged coagulation } \\
\text { profile }\end{array}$ & 16 & 13.3 \\
\hline Preterm labour & 6 & 5 \\
\hline Operative delivery & 67 & 55.8 \\
\hline $\begin{array}{l}\text { Postpartum } \\
\text { haemorrhage }\end{array}$ & 15 & 12.5 \\
\hline
\end{tabular}

Among 120 patients included in the present study $60 \%$ had sleep disturbance due to pruritis. Deranged coagulation profile was seen in $16(13.3 \%)$ patients. $55.8 \%$ of the patients underwent operative delivery. Preterm labour was present in $5 \%$ patients. Incidence of postpartum haemorrhage was $12.5 \%$ (Table 5). Preterm labour was present in $5 \%$ patients. Incidence of postpartum haemorrhage was $12.5 \%$ (Table 5).

Table 6: Distribution of foetal outcome.

\begin{tabular}{|lll|}
\hline Variables & No. of cases & $\%$ \\
\hline Abnormal CTG & 24 & 20 \\
\hline Meconium stained liquor & 56 & 46.6 \\
\hline NICU admission & 44 & 36.6 \\
\hline Perinatal death & 4 & 3.3 \\
\hline Preterm birth & 6 & 5 \\
\hline
\end{tabular}

Table 7: Postpartum resolution after 6weeks of delivery.

\begin{tabular}{|lll|}
\hline Postpartum resolution & No. of cases & $\%$ \\
\hline Normal LFTs & 118 & 98.3 \\
\hline Persisting raised LFTs & 2 & 1.6 \\
\hline
\end{tabular}

During labor an abnormal CTG was noted in $20 \%$ of the cases, while meconium staining of liquor was seen in $46.6 \%$ of the cases. NICU admission was seen in $36.6 \%$ newborns of which perinatal death was present in $3.3 \%$ cases. $5 \%$ patients had preterm birth (Table 6). In present study, in most of the cases normal LFT $(98.3 \%)$ was found after 6 weeks of delivery and symptomatically pruritus relieved. So the disease resolved within 6 weeks of delivery. (Table-7).

\section{DISCUSSION}

The study was conducted to determine maternal outcome in patients with cholestasis of pregnancy.120 patients were studied. Incidence of obstetric cholestasis was $9.3 \%$ in the present study comparable to the study of Kaur et al $(16.75 \%)^{11}$

Majority of the patients were in the age group of 26-30 years $(48.3 \%)$. Brouwers et al also performed similar study on subjects with intrahepatic cholestasis. ${ }^{12}$ In a prospective population-based study by Geenes et al to assess outcome in severe IHCP, mean age was $29.6( \pm 6.3)$ years. Both the studies were in accordance to present study with mean age also similar to population of present study. In a study by Shoballi et al with similar objectives also the age of IHCP subjects was found to be $29.18 \pm 3.54$ and those of the controls was $29.86 \pm 4.37$ years. ${ }^{13}$ Out of 120 patients, $78.3 \%$ delivered at term, $5 \%$ patients had preterm delivery. Our results were consistent with Rasheed et al. ${ }^{14}$

In present study various parameters of liver damage including total bilirubin, direct bilirubin, aspartate aminotransferase, alanine aminotransferase and alkaline phosphatase were found to be significantly elevated. Jaundice was present in $22(18.3 \%)$. SGOT in maximum number of patients $52(43.3 \%)$ were in the range of 100 200, SGPT in maximum patients $54(45 \%)$ were in the range of $0-100$. ALP was in the range of 400-600 in $35 \%$ of the patients (Table 3 ). Although serum bile acids are considered to be very sensitive indicator of obstetric cholestasis, but as bile acid assessment was not available locally, we could not determine the levels in our patients. The above results are consistent with Rasheed et al, they found liver functions deranged in $73.3 \%$ and $22 \%$ had elevated bilirubin. In a study by Brouwers et al, bilirubin and all liver enzymes including AST, ALT, ALP, GGT, and LDH were found to be elevated. Surprisingly these parameters were highest in subjects with moderate severity of IHCP. ${ }^{12}$

In the present study $41.6 \%$ delivered vaginally. Caesarean section was done in $67(55.8 \%)$ patients (Table 4$)$. Out of 67 caesarean sections, 15 were elective because of obstetric indications like malpresentations, previous LSCS, CPD etc and 52 were emergency LSCS. Most of the emergency LSCS were done at $\geq 38$ weeks GA and hence, were due to foetal distress. Foetal distress was more common after 38 weeks of gestation. Instrumental delivery rate was $2.5 \%$. The above result is consistent Ray Alokananda et al, they found LSCS rate to be $31.2 \%$, however, and instrumental delivery rate was $25 \%$ which was higher than in our study. ${ }^{15}$ This study shows $60 \%$ of the patients had sleep disturbance, due to severe pruritus at night. Generalized pruritus more affecting to palm and sole were the cardinal features without skin rash and clinical jaundice. In this study, we treated our patients with topical 
emollients like calamine lotion and oral Ursodeoxycholic Acid (300-1200) in divided doses.

In the present study $13.3 \%$ of the patients had deranged coagulation profile with increased PT or APTT level. Postpartum haemorrhage accounted for $12.5 \%$ in the present study. Dang et al $(29.78 \%)$ and Ray et al (25\%) reported significant increased incidence of $\mathrm{PPH}$, as a result of malabsorption of vitamin $\mathrm{K}$, due to steatorrhoea of cholestasis, leading to coagulation problem. ${ }^{16,17}$ Kenyon $\mathrm{AP}$ et al found a high incidence of PPH in OC patients who did not receive vitamin $\mathrm{K}$ compared to those who did (45\% vs $12 \%) .^{18}$

Abnormal CTG was found in $20 \%$ of the cases in present study. 56 patients had meconium stained liquor during delivery (46.6\%) which was significantly higher. Alsulyman et al also found that risk of meconium passage was higher in the cholestasis group (44.3\% cases vs. $7.6 \%$ of control). ${ }^{19}$ It has been suggested that both foetal distress and increased gut motility by bile acids is the cause of raised incidence of meconium stained liquor.

Perinatal death was found in $6(5 \%)$ cases in the study. This includes one intrauterine foetal death and 5 neonatal deaths. Alsulyman et al also found 2 of 79 patients having intrauterine foetal death in Obstetric Cholestasis group at 36 to 37 weeks of gestation. ${ }^{19}$ In $36.6 \%$ of the cases NICU admission were required in this study. According to Heinonen et al intrahepatic cholestasis increases the need for intensive neonatal care in general population. ${ }^{20}$

In present study, in most of the cases $(98.3 \%)$ normal LFT was found after 6 weeks of delivery and symptomatically pruritus relieved. According to Rasheed et al postnatal resolution of pruritus and liver function test occurred within 5-14 days with a mean of 8 day $\pm 2.52 .^{21}$

\section{CONCLUSION}

Cholestasis of pregnancy is a relatively common cause of hepatic impairment in pregnancy. It has a complex etiology with genetic, hormonal and environmental components. Maximum patients are delivered at 37 to 38 weeks, due to active maternal and foetal surveillance and early intervention to prevent sudden foetal death at late gestation. Maternal morbidity is increased in terms of increased LSCS rates. Neonatal morbidities are mainly due to foetal distress, prematurity, low birth weight and meconium staining of amniotic fluid. Foetal outcomes are improved with a variety of strategies of active management, although the most effective intervention has not currently been established. Ursodeoxycholic acid treatment is associated with marked improvement of symptoms and biochemical abnormalities. Almost all patients have postnatal resolution within 6 weeks of delivery.

\section{ACKNOWLEDGMENTS}

The authors would like to acknowledge the entire staff of the Department of Obstetrics and Gynecology and all the patients who were studied.

\section{Funding: No funding sources}

Conflict of interest: None declared

Ethical approval: The study was approved by the Institutional Ethics Committee

\section{REFERENCES}

1. Lammert F, Marschall HU, Glantz A, Matern S. Intrahepatic cholestasis of pregnancy: molecular pathogenesis, diagnosis and management. J Hepatol. 2000;33:1012-21.

2. Beuers U, Pusl T. Intrahepatic cholestasis of pregnancy--a heterogeneous group of pregnancyrelated disorders. Hepatol. 2006;43:647-9.

3. Kenyon AP, Piercy CN, Girling J, Williamson C, Tribe RM, Shennan AH. Obstetric cholestasis, outcome with active management: a series of 70 cases. BJOG. 2002;109:282-8.

4. Ray A, Tata RJ, Balsara R. Cholestasis of pregnancy. J Obstet Gynecol India. 2005;55:247-50.

5. Nichols AA. Cholestasis of pregnancy: a review of the evidence. J Perinat Neonatal Nurs. 2005;19:217-25.

6. Bacq Y, Sapey T, Brechot MC, Pierre F, Fignon A, Dubois F. Intrahepatic cholestasis of pregnancy: a French prospective study. Hepatol. 1997;26:358-364.

7. Glantz A, Marschall HU, Mattsson LA. Intrahepatic cholestasis of pregnancy: relationships between bile acid levels and fetal complication rates. Hepatol. 2004;40:467-74.

8. Royal College of Obstetricians and Gynaecologists. Obstetric cholestasis. Green-Top Guideline No 43. 2006. Available at https:// www. rcog. org. uk/en/guidelines-research-services/guidelines/gtg43/. Accessed on 12 February.

9. Lammert F, Marschall H, Matern S. Intrahepatic cholestasis of pregnancy. Curr Treat Options Gastroenterol. 2003;6:123-32.

10. Greenes V, Williamson C. Intahepatic cholestasis of pregnancy. World J Gastroenterol. 2009;15(17):204966.

11. Kaur D, Ruchi S. Hepatic disorders in pregnancy. J Obstet Gynaecol India. 2001;51:31-3.

12. Brouwers L, Koster M, Christiaens PG, Kemperman $\mathrm{H}$, Boon J, Evers I, et al. Intrahepatic cholestasis of pregnancy: maternal and fetal outcomes associated with elevated bile acid levels. Am J Obstet Gynecol. 2015;212(1):100.e1-7.

13. Shobaili AH, Hamed H, Robaee AA, Alzolibani A, Amin A, Ahmad S. Obstetrical and fetal outcomes of a new management strategy in patients with intrahepatic cholestasis of pregnancy. Arch Gynecol Obstet. 2010;283(6):1219-25. 
14. Rasheed S, Afghan S, Mazhar SB. Fetomaternal outcome in patients with obstetric cholestasis. Ann. Pak Inst Med Sci. 2009;5(4):211-5.

15. Alokananda R, Rashne TJ, Roshan B. Nature and outcome of pregnancy in obstetric cholestasis. Obstet Gynecol India. 2005;55(3):247-50.

16. Ray A, Tata RJ, Balsara R. Cholestasis of pregnancy. J Obstet Gynecol India. 2005;55:247-50.

17. Dang A, Agarwal N, Bathla S, Sharma N, Balani S. Prevalence of liver disease in pregnancy and its outcome with emphasis on obstetric cholestasis : an Indian scenario. J Obstet Gynecol India. 2010;60(6):413-8.

18. Kenyon AP, Piercy CN, Girling J, Williamson C, Tribe RM, Shennan AH. Obstetric cholestasis, outcome with active management: a series of 70 cases. BJOG. 2002;109:282-8.

19. Alsulyman OM, Ouzounian JG, Castro AM, Goodwin TM. Intrahepatic cholestasis of pregnancy: perinatal outcome associated with expectant management. Am J Obstet Gynecol. 1996;175:957-60.

20. Heinonen S, Kirkinen P. Pregnancy outcome with intrahepatic cholestasis. Obstet Gynecol. 1999;94:189-93.

21. Rasheed S, Afghan S, Mazhar SB. Fetomaternal outcome in patients with obstetric cholestasis. Ann Pak Inst Med Sci. 2009;5(4):211-5.

Cite this article as: Jamwal D, Kour G, Mehta A. Maternal and perinatal outcome in pregnancy complicated by obstetric cholestasis: study from a tertiary care centre in North India. Int J Reprod Contracept Obstet Gynecol 2021;10:2830-4. 